\section{The reproduction in women affected by cooley disease}

\author{
Carlo Pafumi, ${ }^{1}$ Vito Leanza, ${ }^{1}$ Luana Coco, ${ }^{1}$ \\ Stefania Vizzini, ${ }^{1}$ Lilliana Ciotta, ${ }^{1}$ \\ Alessandra Messina, ${ }^{1}$ Gianluca Leanza, ${ }^{1}$ \\ Giuseppe Zarbo, ${ }^{1}$ Alfio D'Agati, ${ }^{1}$ \\ Marco Antonio Palumbo, ${ }^{2}$ \\ Alessandra lemmola, ${ }^{2}$ \\ Ferdinando Antonio Gulino, 2 \\ Maria Cristina Teodoro, ${ }^{2}$ \\ Matthew Attard, ${ }^{3}$ Alina Cristina Plesca, ${ }^{4}$ \\ Catarina Soares, ${ }^{5}$ Nina Kouloubis, ${ }^{6}$ \\ Mayada Chammas ${ }^{7}$ \\ 1Department of Obstetrics and \\ Gynaecology, University of Catania, Italy; \\ 2Department of Assisted Reproduction, \\ University of Catania, Italy; ${ }^{3}$ Faculty of \\ Medicine \& Surgery, University of Malta; \\ ${ }^{4}$ Faculty of Medicine, University of \\ Medicine and Pharmacy GR.T.Popa lasi, \\ Romania; ${ }^{5}$ Faculdade de Medicina da \\ Universidade do Porto, Portugal; \\ 'University of Rotterdam, The \\ Netherlands; ${ }^{7}$ University of Beirut, Israel
}

\section{Abstract}

The health background management and outcomes of 5 pregnancies in 4 women affected by Cooley Disease, from Paediatric Institute of Catania University, are described, considering the preconceptual guidances and cares for such patients. These patients were selected among a group of 100 thalassemic women divided into three subgroups, according to their first and successive menstruation characteristics: i) patients with primitive amenorrhoea, ii) patients with secondary amenorrhoea and iii) patients with normal menstruation. Only one woman, affected by primitive amenorrhoea, needed the induction of ovulation. A precise and detailed pre-pregnancy assessment was effected before each conception. This was constituted by a series of essays, including checks for diabetes and hypothyroidism, for B and C hepatitis and for blood group antibodies. Moreover were evaluated: cardiac function, rubella immunity and transaminases. Other pregnancy monitoring, and cares during labour and delivery were effected according to usual obstetrics practice.

All the women were in labour when she were 38 week pregnant, and the outcome were five healthy babies born at term, weighting between 2600 and $3200 \mathrm{gs}$. The only complication was the Caesarean section. The improvements of current treatments, especially in the management of iron deposits, the prolongation of survival rate, will result in a continuous increase of pregnancies in thalassemic women. Pregnancy is now a real possibility for women affected by such disease. We are furthermore studying the possibility to collect the fetus' umbilical cord blood, after the delivery, to attempt eterologus transplantation to his mother trying to get a complete marrow reconstitution.

\section{Introduction}

Beta Thalassemia major, also called Cooley disease, is a severe transfusion-dependent anaemia which often causes infertility due to the iron deposits in endocrine organs following multiple blood transfusions, needed to care this illness. Gonadal function is impaired in the majority of the patients affected by the homozygous kind of this disease and, especially in females, this is expressed either by a delay in the onset of puberty and menstruation or by secondary amenorrhoea. ${ }^{1}$ The commonest abnormality was hypogonadotropic hypogonadism, but the relatively high prevalence of other endocrine impairments, causing multiple endocrinopathies, is also well recognised. These kind of endocrine complications are directly related to the serum ferritin level. ${ }^{2}$ The aetiology of the above mentioned reproductive disorders is linked to an anatomical and functional disarray of the components of the central nervous system-gonadal axis and the functionality is often impaired at all three levels, i.e. gonads, anterior pituitary and hypothalamus. ${ }^{3-5}$ Up to the end of 1980 there were only some reports of pregnancy in patients with beta Thalassemia major and, only few of them were carried out successfully. ${ }^{6-8}$ Nowadays, thanks to improved paediatric and haematological cares, patients affected by Cooley disease may live on to be forty or fifty, and can enjoy a quite regular standard of life. So that, maintaining a strict therapeutic regimen, women regularly transfused and well chelated can attempt to become pregnant. ${ }^{9-12}$ In this report we describe the evolution and the successful outcome of five at term pregnancies carried out by four women affected by transfusion dependent beta Thalassemia. In reviewing the cares and the complications during and after their pregnancies, we discuss the issue concerning the appropriate preconception counselling of such patients.

\section{Materials and Methods}

For several years we have been following one hundred women affected by transfusion dependent beta Thalassemia major, diagnosed
Correspondence: Prof Carlo Pafumi, Surgery Department, Vittorio Emanuel hopsital, via plebiscito, 95122 Catania, Italy.

Tel +39.0957435541 - Fax +39.095312001.

E-mail:pafumi@unict.it

Key words: beta-thalassemia major, fertility, pregnancy, umbilical cord blood, transplantation, marrow reconstitution.

Acknowledgment: the authors thank Mrs. Valentina Pafumi for manuscript editing.

Received for publication: 1 January 2011. Accepted for publication: 9 March 2011.

This work is licensed under a Creative Commons Attribution 3.0 License (by-nc 3.0).

(C) Copyright C. Pafumi et al., 2011

Licensee PAGEPress, Italy

Hematology Reports 2010; 3:e4

doi:10.4081/hr.2011.e4

when they were between 6 and 8 months old, from the endocrine and reproductive point of view. All of them, who attended the Paediatric Institute of Catania University, were aged between 23 to 29 and received a blood transfusion every $15-20$ days, in order to maintain the haemoglobin concentration above $10 \mathrm{~g} / \mathrm{dL}$; and were in strict desferrioxamine chelating therapy, to avoid excessive iron overloads. The patients have been divided into three groups according to their first and successive menstruation characteristics: i) patients with primitive amenorrhoea, ii) patients with secondary amenorrhoea and iii) patients with normal menstruation. In detail twenty out of these women got married: four were affected by primitive amenorrhoea; six were affected by secondary one and the remaining ten women had normal menstruation. Finally five pregnancies occurred among these twenty women: two among the patients affected respectively by primitive and secondary amenorrhoea, and three were carried out by women with spontaneous menstruation. Considering as to the last three pregnancies, it is remarkable that to this group belonged a woman that had two successful childbirths; so the total number of women we followed was four (Figures 1, 2; Table 1). Average values of ferritin fluctuated between 500 and $1000 \mathrm{ng} / \mathrm{mL}$, and transaminases were between 25 and $100 \mathrm{U} / \mathrm{L}$. All of them were vaccinated against Hepatitis B Virus (HBV), and two were splenectomized, particularly the woman affected by primitive amenorrhoea and one of those with normal periods. Only the woman affected by primitive amenorrhoea needed the induction of ovulation to stimulate her pregnancy. Evaluation of eligibility depends upon the results of a series of examinations regarding hearth, kidneys, thyroid, pancreas and peripheral arteries. Haemosiderotic parenchimal 
deterioration, particularly of the myocardium, makes these patients potentially fragile, while the induction of ovulation may be a hard stress test even for a healthy woman. All the other women had spontaneous and physiological pregnancies. It is finally important to underline that all the partners of these women were negative as regards the thalassemic trait. According to the guidelines previously established by Susan M. Tuck, ${ }^{13}$ a precise and detailed prepregnancy assessment was effected before each conception. This was constituted by a series of clinical and laboratory essays, including checks for diabetes and hypothyroidism, for B and C hepatitis and for blood group antibodies. Cardiac function was assessed symptomatically and by echocardiography and exercise electrocardiography. Rubella immunity was checked and immunisation was given when the woman was found to be susceptible. During pregnancy the monitoring of all these factors continued, including frequent checks of aminotransferase (AST and ALT transaminases). The frequency of blood transfusion had to be increased, the aim being to maintain the pre-transfusion haemoglobin concentration. Desferrioxamine therapy was stopped as soon as pregnancy was diagnosed, because using this drug during gestation could notoriously induce teratogenic effects on the foetus, but the average values of ferritin were just a little higher than the normal range observed. Probably the foetus, due to a particular chelating activity, maintains it during gestation. This therapy was quickly resumed after delivery. ${ }^{14}$ Other pregnancy monitoring, such as for blood pressure, for proteinuria and for fetal growth, and cares during labour and delivery were effected according to usual obstetrics practice and criteria.

\section{Results}

Only one out of the five pregnancies we have followed needed the induction of ovulation. Moreover a same woman carried out two of them. All the women were in labour when she were 38 week pregnant, and the outcome were five healthy babies born at term weighting between 2600 and $3200 \mathrm{gs}$. The caesarean delivery was preferred because of the frequent infertility/sterility that occurs in the natural history of such kind of patients. All of these women were neither HIV positive nor suffered from diabetes, however they were controlled with a diet. All of them reported transaminases raising, but only in one case it was necessary to practice a therapy based on silimarina. Moreover all of them had transfusion-transmitted $\mathrm{C}$ hepatitis prior to pregnancy, but only the one affected by secondary amenorrhoea received curses of interferon two years before embarking on pregnancy, because she showed to be positive for
HCV-RNA. Four out of the babies born were HCV negative, while only one was HCV positive but HCV-RNA negative, so that it was not necessary to prescribe a therapy.

Finally, there were neither cardiac dysfunctions nor kidney diseases reported, or other important affections.

\section{Discussion}

We believe that several ethical and theoretical questions should be evaluated before the observation of technical problems. We should never forget that Beta thalassemic are fragile patients

Table 1. Table expressing the number of married women, the pregnancies, the medium age and the amount of each group, according to a subdivision in three categories Normal menstruation, Secondary Amenorrhoea and Primitive Amenorrhoea - in reference to the total amount of the 100 patients we studied.

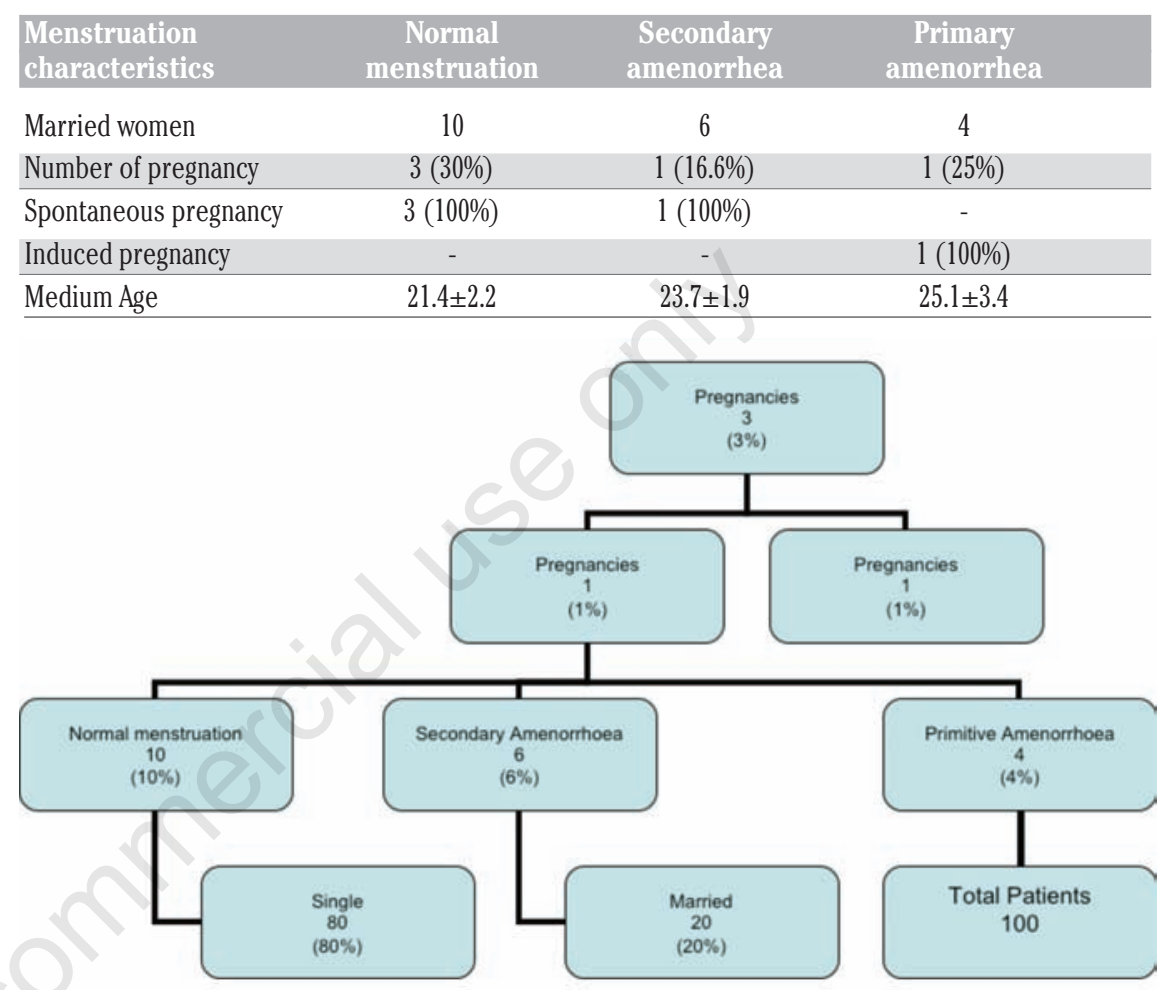

Figure 1. Flow chart representing the distribution of 100 women affected by Beta Thalassemia major subdivided in three categories, according to their menstruation characteristics; considering if they are married or singles, and the number of pregnancies carried out by each group.

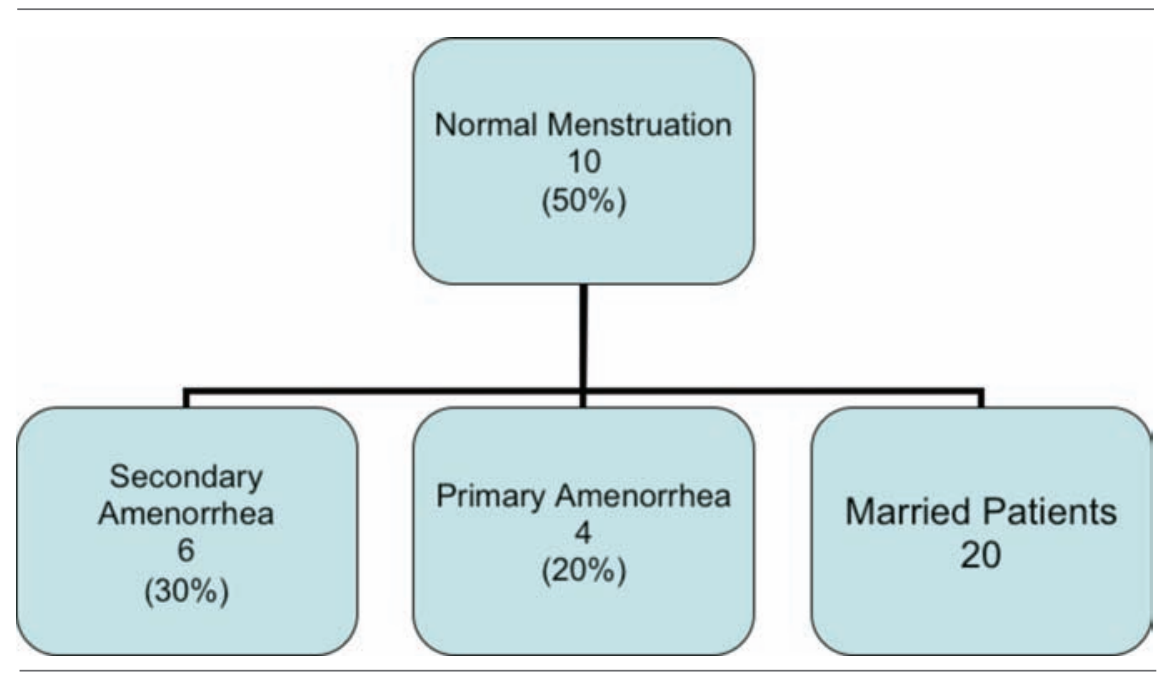

Figure 2. Graphic expressing the percentage of married women in reference to each group in which we subdivided them. 
who are knowingly exposed to a large quantity of risks. It is the doctor's responsibility to decide which patient can afford ovulation induction, when needed, pregnancy and labour, with an acceptable degree of risk, and which are the limits of this risk. As regards the possibility to induce a pregnancy in women affected by such disease, this activity was judged to be perfectly moral from the chatolic point of view by the Center for Bioethics of the Chatolic University of the Sacred Heart, Rome, as it was explained in previous studies regarding this matter. ${ }^{15}$

Finally, the improvements of current conventional treatments, especially in the management of iron deposits, the prolongation of survival rate, and the new approaches to assisted conception, when needed, will result in a continuous increase of pregnancies in women affected by beta Thalassemia. According to the several advances in treatment, occurred from the 1980 s, and to periconceptional counselling - concerning the continuation of pregnancy only in women in good general conditions - intrauterine growth retardation, foetal loss and preterm labour are remarkably reducing.

Concluding we can state that pregnancy is now a real possibility for women affected by Cooley disease that are regularly transfused and well chelated. Pregnancy does not have a

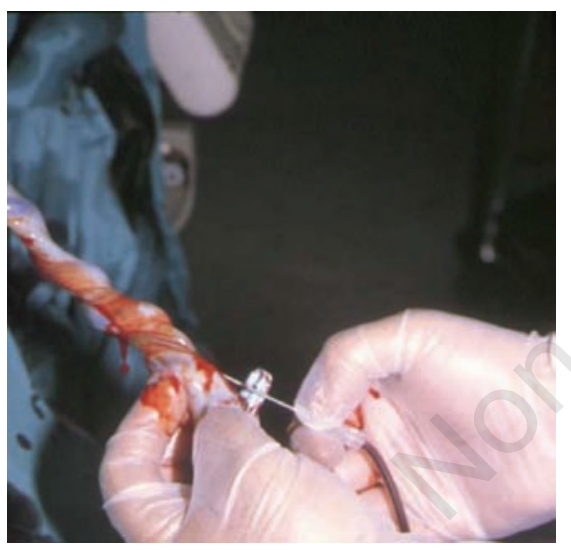

Figure 3. Photograph showing the collection of umbilical cord blood, containing a large quantity of CD34+ stem cells, during a caesarean section, in a thalassemic woman. deleterious effect on the course of their disease, and on the general well being of the foetus. It seems, in fact, that the evaluation of cardiac function, in combination with a low iron load and satisfactory endocrine and hepatic controls can ensure a pregnancy outcome with minimal adverse effects, and not severe obstetric complications except for the high incidence of caesarean sections. ${ }^{16}$

Then it is possible to think about a normal pregnancy without fetal loss, foetus' intrauterine growth retardation and preterm labour, like it is commonly believed. We are furthermore studying the possibility to collect a good quantity of umbilical cord blood of the foetus, after the delivery and before placental detachment, to attempt eterologus transplantation to the mother (Figure 3). ${ }^{17}$ This is to be hoped, especially if the mother is totally HLA-matched or also partially matched with her son, because umbilical cord blood has shown to possess a significant quantity of CD34+ ${ }^{+}$stem cells. These particular haematological stem cells have a great capacity to repopulate the bone marrow and now are being used to intent an alternative approach to several both malignant and not haematological disease. So, by using this procedure, after several laboratory passages of $\mathrm{CD} 34^{+}$recovering, metabolism monitoring, enrichment and clonogenic culture, we aim at trying to get a complete marrow reconstitution of the thalassemic mother, thanks to the help offered by her donor son.

\section{References}

1. Karagiorga-Lagana M. Fertility in Thalassemia: The Greek Experience. J Pediatr Endocrinol Metab 1998;11:945-51.

2. Jensen CE, Tuck SM, Old J, Morris RW, et al. Incidence of endocrine complications and clinical disease severity related to genotype analysis and iron overload in patients with beta-thalassaemia. Eur J Haematol 1997;59:76-81.

3. Vullo C, De Sanctis V, Katz M, et al. Endocrine abnormalities in Thalassemia. Ann Ny Acad Sci 1990;612:293-310.

4. Jensen CE, Tuck SM. Endocrine problems in Beta-Thalassemia major. Contemp Rev
Obstet Gynaecol 1994;6:133-6.

5. Tolis G, Vlachopapadopoulou E, Karydis J. Reproduction health in patients with BetaThalassemia. Curr Opin Pediatr 1996;8: 406-10.

6. Thomas RM, Skalioka AE. Successful pregnancy in transfusion dependent Thalassemia. Arch Dis Child 1980;55:5724.

7. Martin K. Successful pregnancy in beta Thalassemia major. Aus Paediatr J 1983; 19:182-3.

8. Meadows K. A successful pregnancy outcome in transfusion dependent Thalassaemia major. Aus NZ J Obstet Gynaecol 1884;24:43-4.

9. Savona-Ventura C, Bonello F. BetaThalassemia syndromes and pregnancy. Obstet Gynecol Surv 1994;49:129-37.

10. Kumar RM, Rizk DE, Khuranna A. BetaThalassemia major and successful pregnancy. J Reprod Med 1997;42:294-8.

11. Tampakoudis P, Tsatalas C, Mamopoulos M, et al. Transfusion-dependent homozygous beta-Thalassaemia major: successful pregnancy in five cases. Eur J Obstet Reprod Biol 1997;74:127-31.

12. Daskalakis GJ, Papageorgiou IS, Antsaklis AJ, Michalas SK. Pregnancy and homozygous beta thalassaemia major. Br J Obstet Gynaecol 1998;105:1028-32.

13. Tuck SM, Jensen CE, Wonke B, Yardumian A. Pregnancy management and outcomes in women with Thalassemia major. J Paediatr Endocrinol Metab 1998;11:923-8.

14. Vaskaridou E, Konstantopoulos K, Kyriakou D, Loukopoulos D. Deferoxamine treatment during early pregnancy: absence of teratogenicity in two cases. Haematologica 1993;78:183-4.

15. Negri P, Tomasi A, Ricciardelli A, et al. Induzione della ovulazione in paziente beta talassemica omozigote. Medicina e Morale 1991;6:1045-63.

16. Skordis N, Christou S, Koliou M, et al. Fertility in Female Patients with Thalassemia. J Pediatr Endocrinol Metab 1998;11:935-43.

17. Rubinstein P, Carrier C, Scaradavou A, et al. Outcomes among 562 recipients of placental blood transplants from unrelated donors. New Engl J Med 1998;339:1565-77. 